\title{
A Universal Threshold for the Assessment of Load and Output Residuals of Strain-Gage Balance Data
}

\author{
N. Ulbrich ${ }^{\dagger}$ and T. Volden $\ddagger$ \\ Jacobs Technology Inc., Moffett Field, California 94035
}

\begin{abstract}
A new universal residual threshold for the detection of load and gage output residual outliers of wind tunnel strain-gage balance data was developed. The threshold works with both the Iterative and Non-Iterative Methods that are used in the aerospace testing community to analyze and process balance data. It also supports all known load and gage output formats that are traditionally used to describe balance data. The threshold's definition is based on an empirical electrical constant. First, the constant is used to construct a threshold for the assessment of gage output residuals. Then, the related threshold for the assessment of load residuals is obtained by multiplying the empirical electrical constant with the sum of the absolute values of all first partial derivatives of a given load component. The empirical constant equals $2.5 \mathrm{microV} / \mathrm{V}$ for the assessment of balance calibration or check load data residuals. A value of $0.5 \mathrm{microV} / \mathrm{V}$ is recommended for the evaluation of repeat point residuals because, by design, the calculation of these residuals removes errors that are associated with the regression analysis of the data itself. Data from a calibration of a six-component force balance is used to illustrate the application of the new threshold definitions to real-world balance calibration data.
\end{abstract}

\section{Nomenclature}

a $\quad=$ distance between the forward and aft normal force gages of a force balance

$A F \quad=$ axial force

$b \quad=$ distance between the forward and aft side force gages of a force balance

$\mathbf{B}_{\mathbf{1}} \quad=$ square matrix that helps define a load iteration scheme for the Iterative Method

$\mathbf{B}_{\mathbf{2}} \quad=$ auxiliary matrix that helps define a load iteration scheme for the Iterative Method

$c_{0}, c_{1}, \ldots=$ regression coefficients of a gage output that is fitted as a function of the loads

$C_{i} \quad=$ capacity or, alternatively, maximum applied load of a balance

$\mathbf{C}_{1} \quad=$ square matrix that helps define a load iteration scheme for the Iterative Method

$\mathbf{C}_{\mathbf{2}} \quad=$ auxiliary matrix that helps define a load iteration scheme for the Iterative Method

$d_{\circ}, d_{1}, \ldots=$ regression coefficients of a load that is fitted as a function of the gage outputs

$D_{i} \quad=$ maximum recorded output of a gage

$d F_{i} \quad=$ traditional load residual threshold in engineering units (e.g., lbs, in-lbs, N, Nm)

$d F_{i, \%} \quad=$ traditional load residual threshold in percent of capacity or maximum load

$F_{i} \quad=$ known load of a strain-gage balance

$F_{i}{ }^{\prime} \quad=$ predicted least squares approximation of a load of a strain-gage balance

$\mathbf{F} \quad=$ vector of loads that helps define a load iteration scheme for the Iterative Method

$\mathbf{H} \quad=$ rectangular matrix that helps define a load iteration scheme for the Iterative Method

$i \quad=$ index of a gage output or of a load component

$j \quad=$ load iteration step index

$n \quad=$ number of independent gages or number of independent loads of a balance

$N F \quad=$ normal force

$\dagger$ Aerodynamicist, Jacobs Technology Inc.

$\ddagger$ Computer Engineer, Jacobs Technology Inc. 


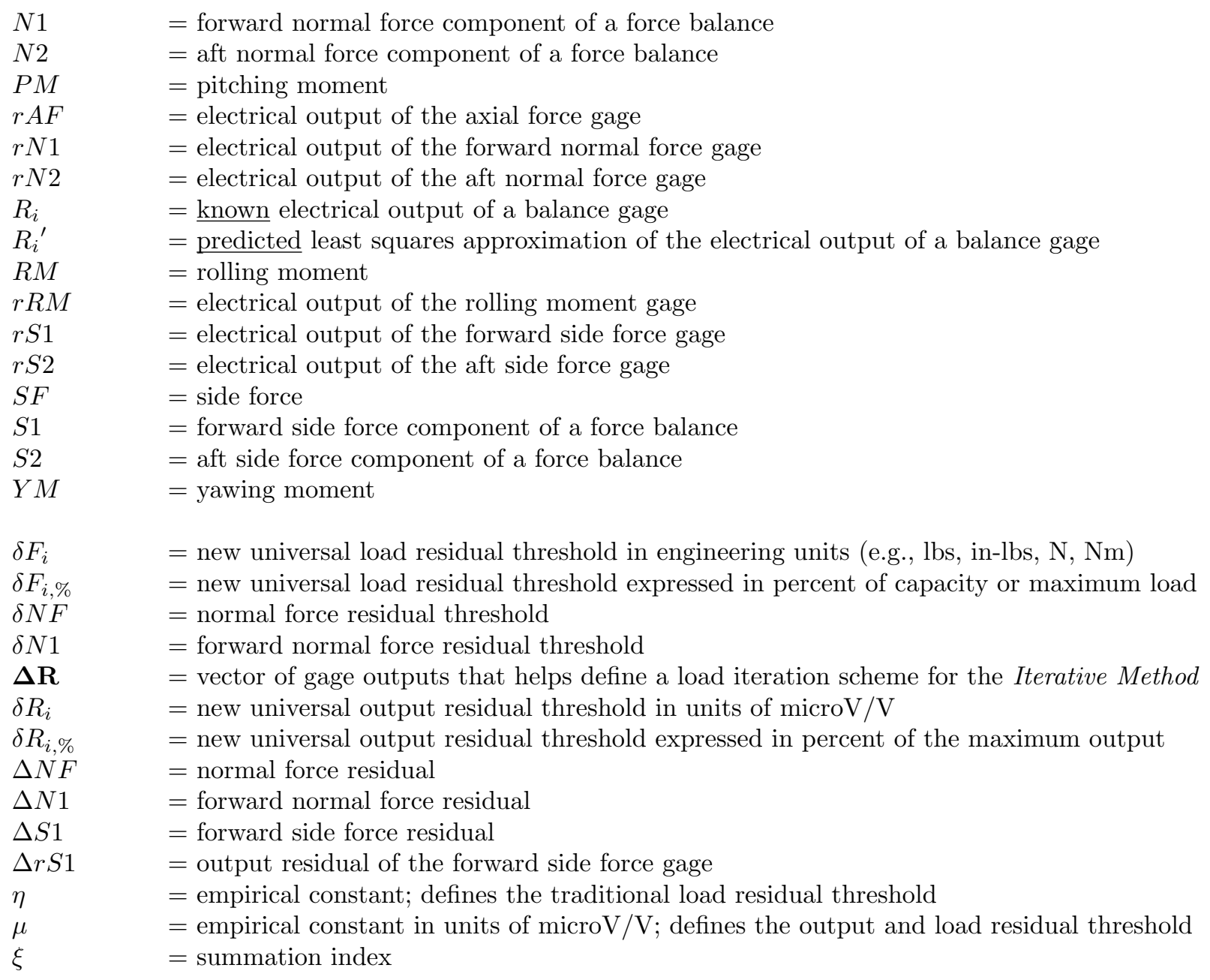

\section{Introduction}

In general, the user of a wind tunnel strain-gage balance should evaluate its expected performance in order to determine if it will meet all accuracy requirements of the planned wind tunnel test. Both load and gage output residuals are used for this purpose. These residuals are defined as the difference between the measured and fitted values of either the loads or the gage outputs. They are a measure of load or output prediction errors that result from imperfections in data acquisition, calibration hardware, and regression analysis of the balance calibration data.

Load residuals are always reported whenever an analyst selects either the Iterative or the Non-Iterative Method for the regression analysis of strain-gage balance data (see Refs. [1] to [3] for descriptions of the two methods). Residuals of the electrical outputs, on the other hand, are only computed as an intermediate result during the application of the Iterative Method as this approach first fits outputs as a function of loads and, afterwards, uses an iteration scheme to predict loads from measured gage outputs.

Another important aspect of the assessment of balance data is the evaluation of residuals of repeat points. This data quality test should be applied to the loads or outputs of the balance because it may help detect calibration process imperfections that need to be addressed. The residuals of repeat points are computed by taking the difference between the predicted load (or measured output) of a repeat point and the arithmetic mean of the loads (or outputs) of the related repeat point set. Afterwards, a suitable threshold may be used to assess if the repeatability, i.e., the precision error, of the loads or outputs meets expectations.

Traditionally, analysts use a small fraction of the capacity of a load component to decide if a load residual meets their accuracy goal, or, if it is a potential outlier that needs to be examined. This threshold 
definition is simple. However, it has a disadvantage: it does not take the load resolution capability of each balance gage into account. Therefore, the authors developed an alternate threshold definition in 2015 that addressed this fundamental shortcoming of the traditional threshold (see Ref. [4]). It was soon recognized that the authors' alternate threshold definition of 2015 has a practical limitation that had to be fixed: it can only be applied if balance data is provided in its "design" format (e.g., force balance in force balance format, or, moment balance in moment balance format). Consequently, the authors developed a more universally applicable residual threshold definition in 2016 that is the topic of the present paper.

First, the traditional threshold definition is briefly reviewed. Afterwards, the new threshold definition of 2016 is introduced. Finally, data from a real-world balance calibration data set is used to illustrate the application of the new threshold.

\section{Traditional Load Residual Threshold}

Analysts often use a small fraction of the capacity of a load component to decide if a load residual meets their accuracy goal, or, if it is a potential outlier that needs to be examined in more detail. The following threshold definition is typically applied

\section{TRADITIONAL LOAD RESIDUAL THRESHOLD (ENGINEERING UNITS)}

$$
\begin{gathered}
d F_{i}= \pm \eta \times C_{i} \quad ; \quad 1 \leq i \leq n \\
\eta=\text { const. }= \begin{cases}0.0025(0.25 \%) \Longrightarrow \text { calibration/check load residuals } \\
0.0010(0.10 \%) \Longrightarrow \text { high precision axial force residuals } \\
0.0005(0.05 \%) \Longrightarrow \text { repeat load residuals }\end{cases} \\
C_{i} \equiv \text { load capacity or, alternatively, maximum applied load }
\end{gathered}
$$

where $\eta$ is a dimensionless empirical constant and $C_{i}$ is the capacity or, alternatively, the maximum applied load of the load component with index $i$. Equation (1b) lists three values of constant $\eta$ that are frequently used in the aerospace testing community. $\Longrightarrow$ The first choice $(0.0025$ or $0.25 \%)$ is the baseline value that helps to evaluate calibration or check load residuals of most balances. $\Longrightarrow$ The second choice $(0.0010$ or $0.10 \%$ ) is used to primarily assess high precision axial force residuals of balances that are used for performance tests. In that case, the "one drag count" accuracy goal of some balance users translates to approximately $0.10 \%$ of the nominal axial force capacity of a well selected balance $(0.10 \%$ of a maximum drag coefficient of $1 / 10$ equals $1 / 10000$, i.e., one drag count, assuming that the maximum drag coefficient occurs when the axial force of the balance is near its capacity). $\Longrightarrow$ The third choice $(0.0005$ or $0.05 \%)$ represents the highest accuracy goal for balance load residuals. It is applied to load differences of repeat points. It is the smallest of the three choices for $\eta$ because the calculation of load differences of related repeat points removes errors associated with the global regression analysis of the balance data.

It is useful to express the traditional threshold defined in Eq. (1a) as a percentage of the capacity as load residuals are often reported in that format. Then, the traditional threshold becomes:

\section{TRADITIONAL LOAD RESIDUAL THRESHOLD (\% OF CAPACITY)}

$$
d F_{i, \%}=\underbrace{d F_{i}}_{E q .(1 a)} \times \frac{100 \%}{C_{i}}= \pm \eta \times 100 \% \quad ; \quad 1 \leq i \leq n
$$

Equation (2) highlights another important characteristic of the traditional load residual threshold def- 
inition: the traditional threshold is the same for all balance gages if it is expressed as a percentage of the load capacity. This conclusion can also be expressed as follows:

$$
d F_{i, \%}=f(\eta) \text { and } \eta=\text { const. } \quad \Longrightarrow \quad d F_{1, \%}=\ldots=d F_{i, \%}=\ldots=d F_{n, \%}
$$

The threshold characteristic described in Eq. (3) has two practical advantages: (i) the threshold definition is easy to apply because a fixed percentage of the load capacity is used for all load components of the balance; (ii) the constant $\eta$ is dimensionless. Therefore, output residuals could also be evaluated by using Eq. (1a) if the capacity of a load component is simply replaced by the maximum measured output of a gage. These two practical advantages, however, could also be interpreted as a disadvantage: the traditional threshold definition does not take into account if an individual gage was optimally sized for the load range that it measures (for example, a correctly matched gage of a force balance has $\approx 1000$ microV/V output at load capacity). Consequently, the traditional threshold definition could lead an analyst into believing that a load residual exceeds the threshold even though, in reality, the residual is within bounds that are dictated by the resolution limitations of the balance gage.

The authors developed an alternate load residual threshold definition in 2015 that addresses some of the above mentioned shortcomings of the traditional threshold (Ref. [4]). Further improvements of the alternate threshold definition were made in 2016. These modifications resulted in a more universally applicable residual threshold definition that is discussed in detail in the next section of the paper.

\section{Universal Gage Output and Load Residual Threshold}

The authors made several assumptions in order to define a more universally applicable residual threshold that (i) would work for both outputs and loads, (ii) would no longer be limited to a specific balance data format, and (iii) would include information about the load resolution capability of each individual balance bridge. Theoretically, the connection between the residual threshold of the outputs and the residual threshold of the loads is possible as the number of independent gage output measurements must always match the number of independently applied load components (see Ref. [5] for a detailed discussion of this basic balance design requirement). Then, a "unique" mapping between gage outputs and loads can be established as a given set of measured outputs only describes one specific combination of load values. This fundamental relationship between the number of strain-gages, i.e., independent strain-gage measurements, and the number of applied load components can be summarized as follows:

$$
\text { number of gages (bridges) } \equiv \text { number of load components }=n
$$

The balance characteristic described in Eq. (4) also means that it should be possible to somehow relate an empirical threshold used for the outputs of a strain-gage balance directly to the empirical threshold of the loads if the "mapping" function between the output "space" and load "space" is known (see Ref. [5]).

The authors' definition of the universal residual threshold for both outputs and loads can be better understood if the regression models of the gage outputs are reviewed in more detail. These regression models are needed by the Iterative Method as this approach first fits outputs as a function of loads. The regression model of the outputs can simply be written as

$$
\underbrace{R_{i}}_{\text {known }} \approx \underbrace{R_{i}{ }^{\prime}}_{\text {predicted }}=\underbrace{c_{0}(i)}_{\text {intercept }}+\underbrace{c_{1}(i) \cdot F_{1}+c_{2}(i) \cdot F_{2}+\cdots+c_{n}(i) \cdot F_{n}}_{\text {linear terms }}+\cdots ; 1 \leq i \leq n
$$

where $R_{i}$ is the known measured electrical output of the gage with index $i, R_{i}{ }^{\prime}$ is the predicted least squares approximation of the measured electrical output, $F_{1}, F_{2}, \ldots F_{n}$ are the loads of the balance, and $c_{\circ}, c_{1}, \ldots$ are the regression coefficients of the regression model of the output (see also Ref. [1], Eqs. (3.1.2) and (3.1.3)). The coefficients are obtained by fitting the outputs of the balance gage as a function of the intercept and the regressors that are obtained from the loads. The difference between the known output $R_{i}$ and the predicted output $R_{i}{ }^{\prime}$ of Eq. (5) is the output residual. This residual can simply be evaluated by using an empirical electrical constant. In other words, a data point is assumed to meet expectations if its residual is below the empirical constant. Therefore, the following universal output residual threshold can be defined as 


\section{UNIVERSAL OUTPUT RESIDUAL THRESHOLD}

$$
\begin{gathered}
\delta R_{i}= \pm \mu \quad ; \quad 1 \leq i \leq n \\
\mu=\text { const. }=\left\{\begin{array}{l}
2.5 \text { micro } V / V \Longrightarrow \text { used for fitted gage outputs } \\
0.5 \text { microV } / V \Longrightarrow \text { used for outputs of repeat points }
\end{array}\right.
\end{gathered}
$$

where $\mu$ is an empirical electrical constant. The output residual threshold definition given in Eq. (6a) is "universal" in nature because it assumes that a measured voltage of a gage (bridge) is divided by its excitation voltage. It is often useful to express the output residual threshold as a percentage of the maximum recorded electrical output of the gage. Then, the output residual threshold can be written as

\section{OUTPUT RESIDUAL THRESHOLD (\% OF MAXIMUM OUTPUT)}

$$
\begin{gathered}
\delta R_{i, \%}= \pm \mu \times \frac{100 \%}{D_{i}} \quad ; \quad 1 \leq i \leq n \\
D_{i} \equiv \text { maximum recorded output of gage in units of microV } / V
\end{gathered}
$$

where $D_{i}$ is the maximum recorded output of the gage. - It is also possible to fit balance loads directly as a function of the measured gage outputs if the Non-Iterative Method is applied to the data. Then, the regression model of a load can be written as

$$
\underbrace{F_{i}}_{\text {known }} \approx \underbrace{F_{i}^{\prime}}_{\text {predicted }}=\underbrace{d_{\circ}(i)}_{\text {intercept }}+\underbrace{d_{1}(i) \cdot R_{1}+\cdots+d_{n}(i) \cdot R_{n}}_{\text {linear terms }}+\cdots ; 1 \leq i \leq n
$$

where $F_{i}$ is the known load with index $i, F_{i}{ }^{\prime}$ is the predicted least squares approximation of the load, $R_{1}, \ldots, R_{n}$ are the independently measured outputs of the balance, and $d_{\circ}, d_{1}, \ldots$ are the regression coefficients of the regression model of the load (see also the discussion of the Non-Iterative Method in Ref. [3]). The coefficients are obtained by fitting the load as a function of the intercept and the regressors that are obtained from the outputs. The difference between the known load $F_{i}$ and the predicted load $F_{i}{ }^{\prime}$ of Eq. (8) is the load residual.

A "conservative" upper bound of the load residuals can be defined by using the absolute values of the linear coefficients of the load's regression model and the absolute value of some acceptable output variation. Absolute values of the coefficients and the acceptable output variation must be used in this context because all output measurements are assumed to be perfectly independent (i.e., the sign of the output variation of the different gages is assumed to be unknown). Then, we get the following inequality:

$$
\text { conservative upper bound } \Longrightarrow \delta F_{i} \leq\left|d_{1}(i)\right| \cdot\left|\delta R_{1}\right|+\cdots+\left|d_{n}(i)\right| \cdot\left|\delta R_{n}\right|=\sum_{\xi=1}^{n}\left|d_{\xi}(i)\right| \cdot\left|\delta R_{\xi}\right|
$$

It is reasonable to assume that the acceptable output variation equals the empirical constant $\mu$ that was introduced in Eq. (6b) above. Then, we get:

$$
\left|\delta R_{1}\right| \approx\left|\delta R_{2}\right| \approx \cdots \approx\left|\delta R_{n}\right| \approx \text { const. } \approx \mu
$$


In addition, we know that the coefficients of the linear terms of Eq. (8) are least squares approximations of the first partial derivatives of the load with respect to all independent output measurements. This conclusion can be expressed as follows:

$$
\left|d_{\xi}(i)\right| \approx\left|\frac{\partial F_{i}}{\partial R_{\xi}}\right| \quad ; \quad 1 \leq \xi \leq n
$$

Finally, after using the right-hand sides of Eqs. $(10 a)$ and (10b) to simplify the summation term on the right-hand side of Eq. (9), we get the following expression for a universal load residual threshold:

\section{UNIVERSAL LOAD RESIDUAL THRESHOLD (ENGINEERING UNITS)}

$$
\begin{gathered}
\delta F_{i}= \pm \mu \times \sum_{\xi=1}^{n}\left|\frac{\partial F_{i}}{\partial R_{\xi}}\right| ; 1 \leq i \leq n \\
\mu=\left\{\begin{array}{l}
2.5 \text { micro } V / V \Longrightarrow \text { used for calibration or check load residuals } \\
0.5 \text { microV } / V \Longrightarrow \text { used for load residuals of repeat points }
\end{array}\right.
\end{gathered}
$$

The load residual threshold given in Eq. (11a) is "universal" because (i) the first derivatives of a load with respect to all outputs can always be determined and (ii) the empirical constant is independent of the excitation voltage of the balance. It is important to emphasize that the empirical constant $\mu$ is common to the definition of the universal output and load residual threshold. In other words, it establishes a direct connection between the two thresholds. In addition, only two choices for the constant $\mu$ are needed, i.e., $2.5 \mathrm{microV} / \mathrm{V}$ and 0.5 microV/V, because information about the sensitivity of each balance gage is indirectly contained in the partial derivatives of Eq. (11a). Consequently, the threshold for the general assessment of the axial force residuals of a balance specifically sized for performance tests is correctly determined using $2.5 \mathrm{microV} / \mathrm{V}$ because such a balance must have, by design, a relatively high axial gage sensitivity.

Again, it is useful to express the universal load residual threshold given in Eq. (11a) as a percentage of the load capacity or, alternatively, as a percentage of the maximum applied load. Then, we get:

\section{UNIVERSAL LOAD RESIDUAL THRESHOLD (\% OF CAPACITY)}

$$
\begin{gathered}
\delta F_{i, \%}=\underbrace{\delta F_{i}}_{\text {Eq.(11a) }} \times \frac{100 \%}{C_{i}} \quad ; \quad 1 \leq i \leq n \\
C_{i} \equiv \text { load capacity or, alternatively, maximum applied load }
\end{gathered}
$$

The new load residual threshold has an important property when compared with the traditional threshold that is described in Eq. (2): the universal threshold is no longer identical for all load components if it is expressed as a percentage of the capacity. This conclusion can also be summarized as follows:

$$
\delta F_{1, \%} \neq \ldots \neq \delta F_{i, \%} \neq \ldots \neq \delta F_{n, \%}
$$

In the next section it is explained how approximations of the first partial derivatives of the loads may be obtained for the different analysis approaches that are used in the aerospace testing community.

\section{Identification of First Partial Derivatives}

It is critical for the application of the new universal load residual threshold that estimates of all first partial derivatives of the load are obtained. The Non-Iterative Method directly supplies those values because 
the linear coefficients of the regression model of the load are least squares approximations of the first partial derivatives (see Eq. (8)). This conclusion can be expressed as follows:

\section{NON-ITERATIVE METHOD}

$$
\begin{array}{r}
\left|\frac{\partial F_{i}}{\partial R_{\xi}}\right| \approx \text { absolute value of coefficient } d_{\xi}(i) \\
d_{\xi}(i) \equiv \text { coefficient with index }\{\xi\} \text { from regression model of the load with index }\{i\}
\end{array}
$$

The identification of numerical approximations of the first partial derivatives is also possible if an analyst chooses to use the Iterative Method for the analysis of balance data (see Refs. [1], [2], and [3] for more details on the Iterative Method). This analysis approach first fits the outputs as a function of the loads. Afterwards, a load iteration scheme is constructed from the regression models of the outputs so that loads can be predicted from outputs during a wind tunnel test. Two load iteration equation choices, i.e., Load Iteration Method $A$ and Load Iteration Method B, are available if the Iterative Method is used. They can be summarized by the following two iteration equations

$$
\begin{aligned}
& \underbrace{\text { Load Iteration Method } A}_{\text {method recommended in Ref. [1] }} \Longrightarrow \mathbf{F}_{\mathbf{j}}=\underbrace{\left[\mathbf{C}_{\mathbf{1}}^{-1} \boldsymbol{\Delta} \mathbf{R}\right]}_{\text {constant }}-\underbrace{\left[\mathbf{C}_{\mathbf{1}}^{-1} \mathbf{C}_{\mathbf{2}}\right] \cdot \mathbf{H}_{\mathbf{j}-\mathbf{1}}}_{\text {changes for each iteration step }} \\
& \underbrace{\text { Load Iteration Method } B}_{\text {derived in the appendix of Ref. [2] }} \Longrightarrow \mathbf{F}_{\mathbf{j}}=\underbrace{\left[\mathbf{B}_{\mathbf{1}}^{-1} \boldsymbol{\Delta} \mathbf{R}\right]}_{\text {constant }}-\underbrace{\left[\mathbf{B}_{\mathbf{1}}^{-1} \mathbf{B}_{\mathbf{2}}\right] \cdot \mathbf{F}_{\mathbf{j}-\mathbf{1}}-\left[\mathbf{B}_{\mathbf{1}}^{-1} \mathbf{C}_{\mathbf{2}}\right] \cdot \mathbf{H}_{\mathbf{j}-\mathbf{1}}}_{\text {changes for each iteration step }}
\end{aligned}
$$

where $\mathbf{j}$ is the load iteration step index. The coefficients of the inverse matrices used in the above equations (i.e., inverse matrix $\mathbf{C}_{\mathbf{1}}^{-1}$ for Load Iteration Method $A$, or, inverse matrix $\mathbf{B}_{\mathbf{1}}^{-1}$ for Load Iteration Method B) are the desired least squares approximations of the first partial derivatives of each load component. This conclusion can be summarized as follows:

\section{ITERATIVE METHOD}

$$
\left|\frac{\partial F_{i}}{\partial R_{\xi}}\right| \approx \text { absolute value of coefficient of row }\{i\} \text { and column }\{\xi\} \text { of } \mathbf{C}_{\mathbf{1}}^{-\mathbf{1}} \text { or } \mathbf{B}_{\mathbf{1}}^{-\mathbf{1}}
$$

It is important to point out that, by design, all off-diagonal coefficients of matrix $\mathbf{B}_{\mathbf{1}}^{-1}$ are zero. Therefore, the residual threshold obtained from the coefficients of matrix $\mathbf{B}_{1}^{-1}$ will always be smaller than the alternate residual threshold obtained from the coefficients of matrix $\mathbf{C}_{\mathbf{1}}^{-1}$. - In the next section of the paper results of the application of the universal residual thresholds to calibration data of NASA's MC60E six-component force balance are discussed.

\section{Discussion of Example}

\section{A. General Remarks}

Calibration data of a strain-gage balance was selected to illustrate the application of the new universal gage output and load residual threshold. The chosen balance is NASA's MC60E six-component balance. The MC60E is a 2.0 inch diameter force balance. Table 1 below lists capacities of the MC60E balance in 
force balance format. The balance was calibrated in 2008 using Triumph Aerospace's Automatic Balance Calibration System (ABCS). The final calibration data set consisted of 1904 loadings (no repeat loads were applied during the calibration).

Table 1: Load capacities of NASA's MC60E six-component force balance.

\begin{tabular}{|c|c|c|c|c|c|}
\hline$N 1, \mathrm{lbs}$ & $N 2, \mathrm{lbs}$ & $S 1, \mathrm{lbs}$ & $S 2, \mathrm{lbs}$ & $R M$, in-lbs & $A F, \mathrm{lbs}$ \\
\hline \hline 2,500 & 2,500 & 1,250 & 1,250 & 5,000 & 700 \\
\hline
\end{tabular}

The analysis of the selected balance calibration data set was performed by using NASA's BALFIT regression analysis tool (see Ref. [6]). The data was analyzed by applying both the Iterative Method and the Non-Iterative Method so that results for both approaches could directly be compared. Table 2 below summarizes eight specific cases that were investigated by using both the traditional and the new universal residual threshold definition.

Table 2: Description of analysis cases and results for the MC60E calibration data.

\begin{tabular}{|c|c|c|c|c|c|c|c|}
\hline $\begin{array}{c}\text { Case } \\
\text { No. }\end{array}$ & $\begin{array}{c}\text { Variable } \\
\text { Symbol }\end{array}$ & $\begin{array}{c}\text { Variable } \\
\text { Type }\end{array}$ & $\begin{array}{c}\text { Analysis } \\
\text { Approach }\end{array}$ & $\begin{array}{c}\text { Thres. } \\
\text { Type }\end{array}$ & $\begin{array}{c}\text { Thres. } \\
\text { Value }\end{array}$ & Std. Dev. & Figure \\
\hline \hline 1 & $S 1$ & load & Iterative (A) & trad. & $\pm 0.250 \%$ & $0.1262 \%$ & Fig. 1 \\
2 & $S 1$ & load & Iterative (A) & univ. & $\pm 0.487 \%$ & $0.1262 \%$ & Fig. 2 \\
3 & $r S 1$ & output & Iterative (A) & univ. & $\pm 0.416 \%$ & $0.1224 \%$ & Fig. 3 \\
4 & $N 1$ & load & Iterative (A) & univ. & $\pm 0.254 \%$ & $0.0737 \%$ & Fig. 4 \\
5 & $N 1$ & load & Iterative (B) & univ. & $\pm 0.236 \%$ & $0.0737 \%$ & Fig. 5 \\
6 & $N F$ & load & Iterative (A) & univ. & $\pm 0.263 \%$ & $0.0376 \%$ & Fig. 6 \\
7 & $N 1$ & load & Non-Iterative & univ. & $\pm 0.254 \%$ & $0.0740 \%$ & Fig. 7 \\
8 & $N F$ & load & Non-Iterative & univ. & $\pm 0.263 \%$ & $0.0368 \%$ & Fig. 8 \\
\hline
\end{tabular}

The outputs of the MC60E balance have no "bi-directional" characteristics. Consequently, no absolute value terms were needed in any of its regression models. In addition, no term reduction of the regression models was performed during the data analysis because an initial analysis of the calibration data showed no significant near-linear dependencies between terms of the regression models. Therefore, a regression model of either an output or a load always consisted of a total of twenty-eight terms (intercept, six linear terms, six quadratic terms, and fifteen cross-product terms). In the next section of the paper the eight cases listed in Table 2 above are discussed in more detail.

\section{B. Discussion of Analysis Cases for the Iterative Method}

First, the Iterative Method was applied to the calibration data. Figure 1 below shows load residuals of the forward side force component $S 1$ if they are assessed by using the traditional residual threshold of $\pm 0.25 \%$ of capacity (see Eq. (2)).

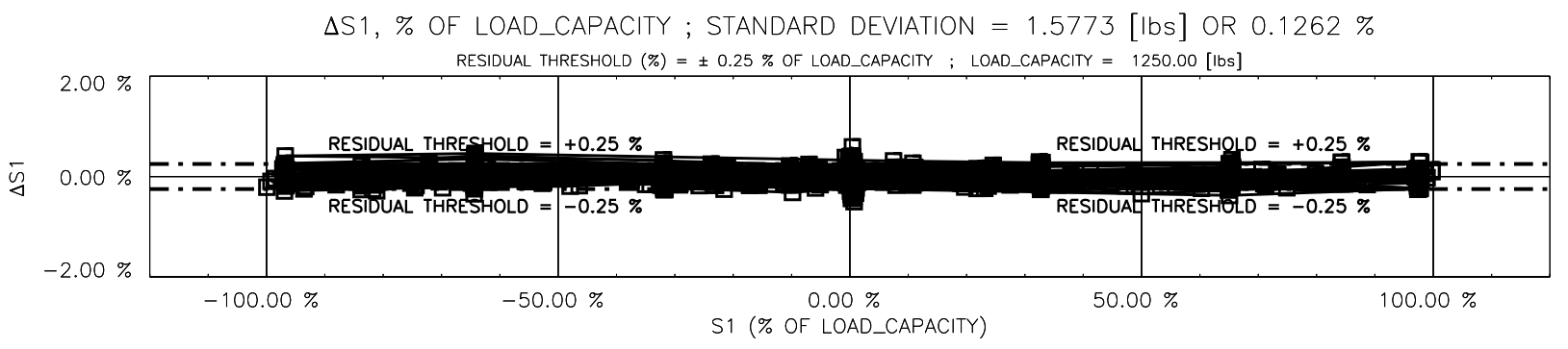

Fig. 1 Load residuals of the forward side force $S 1$ of the MC60E calibration data after having been assessed using the traditional load residual threshold defined in Eq. (2). 
Figure 2 below shows residuals of the same load component if they are assessed by applying the new universal load residual threshold definition. In this case, the residual threshold was computed by using the following equation with $\mu=2.5 \mathrm{microV} / V$ (see also Eq. (12)):

$$
\begin{aligned}
\delta S 1(\%) & = \pm \mu \times[\left|\frac{\partial S 1}{\partial r N 1}\right|+\cdots+\underbrace{\left|\frac{\partial S 1}{\partial r S 1}\right|}_{\text {dominant }}+\cdots+\left|\frac{\partial S 1}{\partial r A F}\right|] \times \frac{100 \%}{\text { capacity }} \\
& = \pm 2.5 \text { microV } / V \times[0.1026+\cdots+2.1807+\cdots+0.0996] \times \frac{100 \%}{1250 \text { lbs }} \\
& = \pm 0.487 \%
\end{aligned}
$$

The threshold value for the forward side force $S 1$ equals $\pm 0.487 \%$ of capacity. Consequently, fewer data points are potential "outliers" if the new instead of the traditional residual threshold is applied.

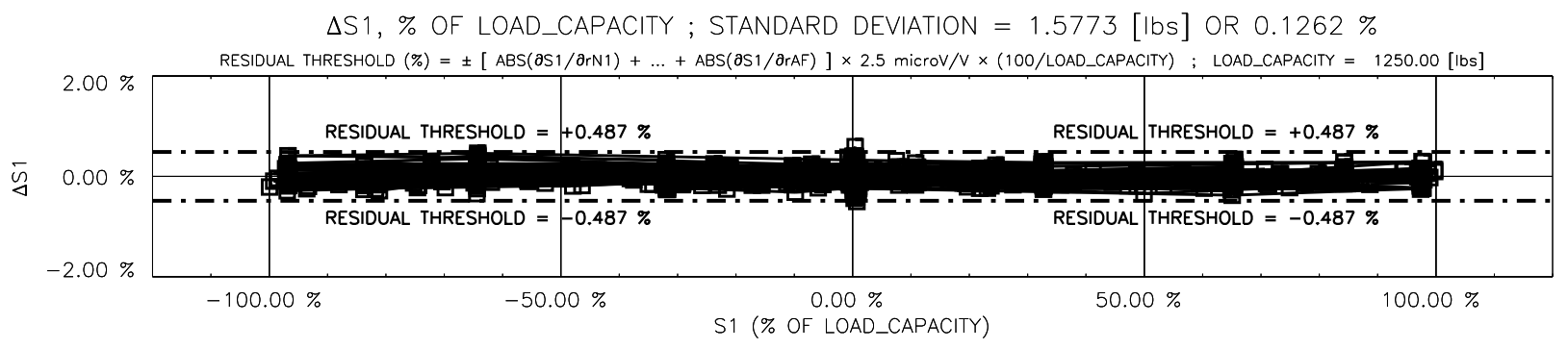

Fig. 2 Load residuals of the forward side force $S 1$ of the MC60E calibration data after having been assessed using the new universal load residual threshold defined in Eq. (12).

This significant difference between traditional and new residual threshold can be explained by the built-in characteristics of the forward side force gage. The reader has to remember that the traditional threshold of $\pm 0.25 \%$ works best for a well matched gage that has a maximum expected output at capacity of $\approx 1000 \mathrm{microV} / \mathrm{V}$. However, the forward side force gage of the MC60E balance only has a maximum expected output of $\approx 600 \mathrm{microV} / \mathrm{V}$. In other words, the forward side force gage has a lower than expected sensitivity. Therefore, the magnitude of the first partial derivative $\partial S 1 / \partial r S 1$ is larger than it would have been if the forward side force gage would have been sized to generate $\approx 1000 \mathrm{microV} / \mathrm{V}$ of output at capacity.

Figure 3 below shows output residuals of the forward side force gage output $(r S 1)$ of the calibration data after having been assessed using the new universal output threshold which is given by the following equation with $\mu=2.5 \mathrm{micro} V / V$ (see also Eq. (7)):

$$
\delta r S 1(\%)= \pm \mu \times \frac{100 \%}{M A X(\text { output })}= \pm 2.5 \mathrm{microV} / \mathrm{V} \times \frac{100 \%}{600.28 \mathrm{microV} / \mathrm{V}}= \pm 0.416 \%
$$

Now, the output residual threshold equals $\pm 0.416 \%$ of the maximum output of the forward side force gage.

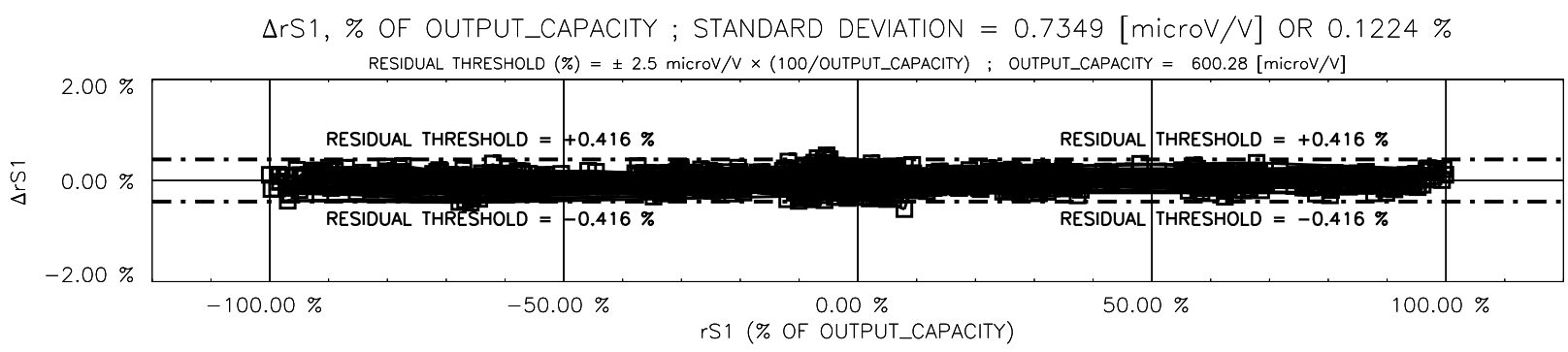

Fig. 3 Output residuals of the forward side force gage $r S 1$ of the MC60E calibration data after having been assessed using the new output residual threshold defined in Eq. (7). 
Again, similar to the result for the universal load residual threshold (Fig. 2), the output residuals shown in Fig. 3 are mostly within the bounds of the universal output threshold.

In the next step, the analysis compares results for the new universal load residual threshold definition after the Iterative Method is used for the data analysis in combination with either Load Iteration Method $A$ or Load Iteration Method B. This time, residuals of the forward normal force $N 1$ were investigated. Figure 4 below shows the load residuals of $N 1$ after Load Iteration Method $A$ is applied. In this case, the residual threshold equals $\pm 0.254 \%$.

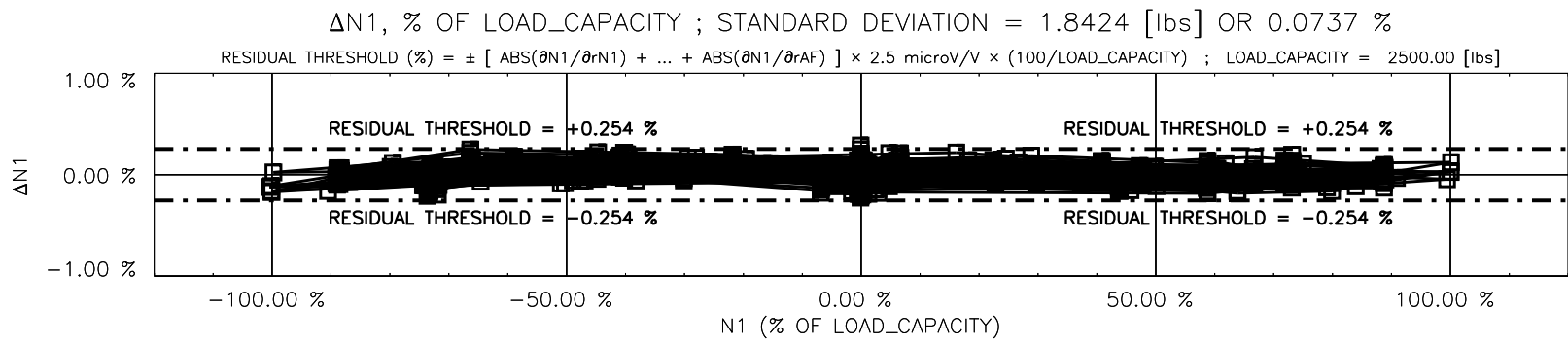

Fig. 4 Load residuals of the forward normal force $N 1$ of the MC60E calibration data after having been processed in force balance format using the Iterative Method \& Load Iteration Method A.

Figure 5 shows the load residual of of $N 1$ after Load Iteration Method B is applied. Now, the residual threshold equals $\pm 0.236 \%$.

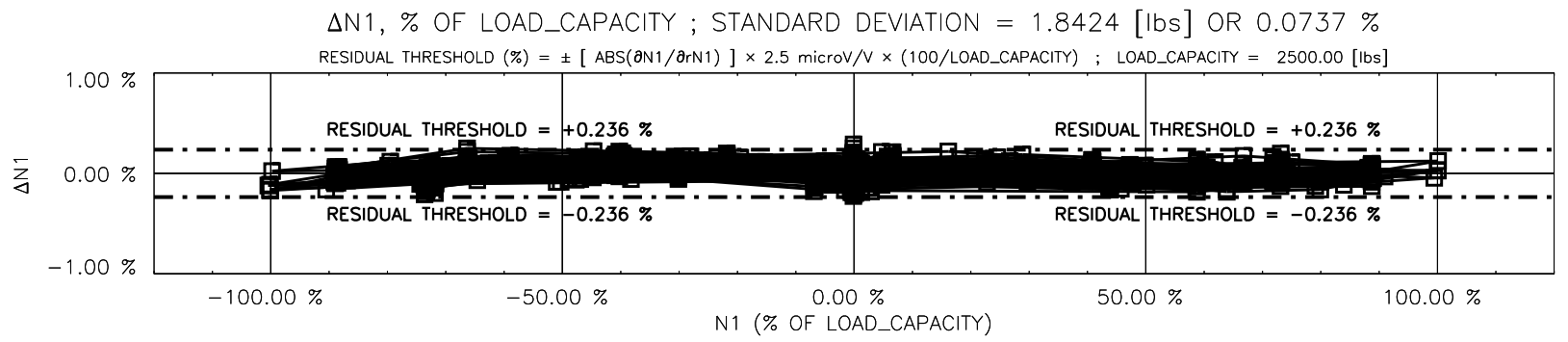

Fig. 5 Load residuals of the forward normal force $N 1$ of the MC60E calibration data after having been processed in force balance format using the Iterative Method \& Load Iteration Method B.

The small reduction of the residual threshold can be explained by the fact that, as mentioned in an earlier section, all off-diagonal coefficients of matrix $\mathbf{B}_{1}^{-1}$ are zero. Therefore, the residual threshold obtained after applying Load Iteration Method B is always smaller than the corresponding threshold for Load Iteration Method $A$ because only approximations of the single most important partial derivative of a load component can be found in matrix $\mathbf{B}_{1}^{-1}$.

Sometimes, loads of a force balance are transformed from force balance to direct-read format before an analysis of the balance calibration data takes place. The universal load residual threshold definition can still successfully be applied in that situation. As an example, residuals of the transformed normal force $N F$ of the MC60E calibration data set were examined. The load transformations from force balance to direct-read format were performed as outlined in Ref. [7]. Then, we get the following relationships

$$
\begin{aligned}
N F & =N 1+N 2 \\
P M & =(N 1-N 2) \cdot(a / 2) \\
S F & =S 1+S 2 \\
Y M & =(S 1-S 2) \cdot(b / 2)
\end{aligned}
$$

where " $a$ " equals the distance of the two normal force gages and " $b$ " equals the distance of the two side force gages. In addition, the transformed capacity of the normal force at zero pitching moment was computed

American Institute of Aeronautics and Astronautics 
to be 5000 lbs (it is the sum of the individual capacities of the forward and aft normal force components). Afterwards, the data was analyzed by applying the Iterative Method in combination with Load Iteration Method $A$. The residual threshold for the transformed normal force $N F$ was computed by using the following equation with $\mu=2.5 \mathrm{microV} / \mathrm{V}$ as input (see also Eq. (12)):

$$
\begin{aligned}
\delta N F(\%) & = \pm \mu \times[\underbrace{\left|\frac{\partial N F}{\partial r N 1}\right|}_{\text {dominant }}+\underbrace{\frac{\partial N F}{\partial r N 2} \mid}_{\text {dominant }}+\cdots+\left|\frac{\partial N F}{\partial r A F}\right|] \times \frac{100 \%}{\text { capacity }} \\
& = \pm 2.5 \text { microV } / V \times[2.1988+2.3067+\cdots+0.1679] \times \frac{100 \%}{5000 \text { lbs }} \\
& = \pm 0.263 \%
\end{aligned}
$$

Figure 6 shows residuals of the transformed normal force $N F$. The residual threshold value for the normal force $N F$ becomes $\pm 0.263 \%$ of capacity.

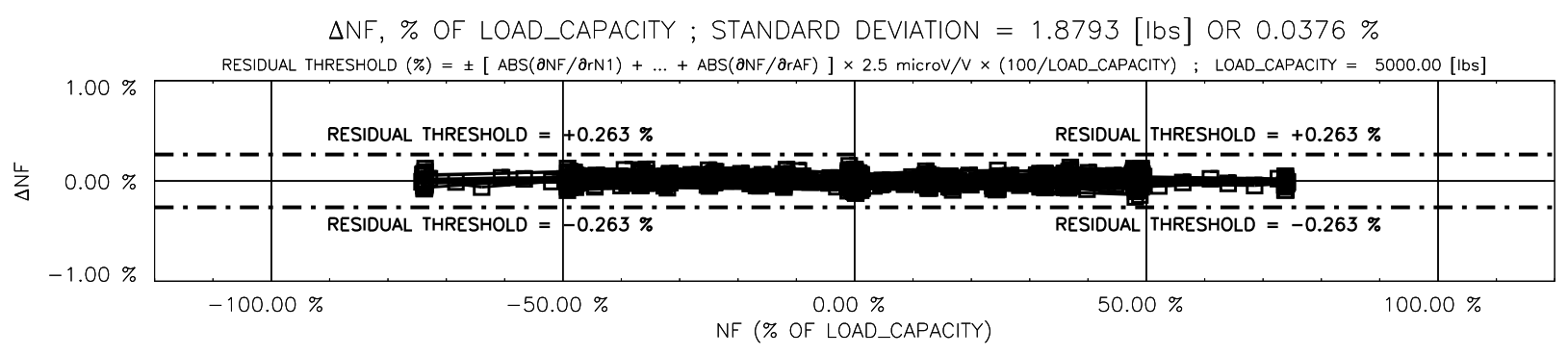

Fig. 6 Load residuals of the total normal force $N F$ of the MC60E calibration data after having been processed in direct-read format using the Iterative Method \& Load Iteration Method A.

It is important to note that two of the six partial derivatives of the total normal force appear to dominate the calculation of the residual threshold (see Eq. (20) above). They are $\partial N F / \partial r N 1$ and $\partial N F / \partial r N 2$. This result is expected because both the forward and aft normal force gage show a significant output change whenever a normal force is applied to the balance. Overall, it can be seen in Fig. 6 that all residuals of the transformed normal force are within the limits of the residual threshold. In other words, the transformed normal force of the MC60E appears to perform as intended.

\section{Discussion of Analysis Cases for the Non-Iterative Method}

It is also interesting to compare residual plots and thresholds of the forward normal force $N 1$ and the total normal force $N F$ with corresponding results that are obtained after applying the Non-Iterative Method. Figure 7 shows results for the forward normal force $N 1$ after the Non-Iterative Method was used to process the data. In this case, the residual threshold is computed to be $\pm 0.254 \%$.

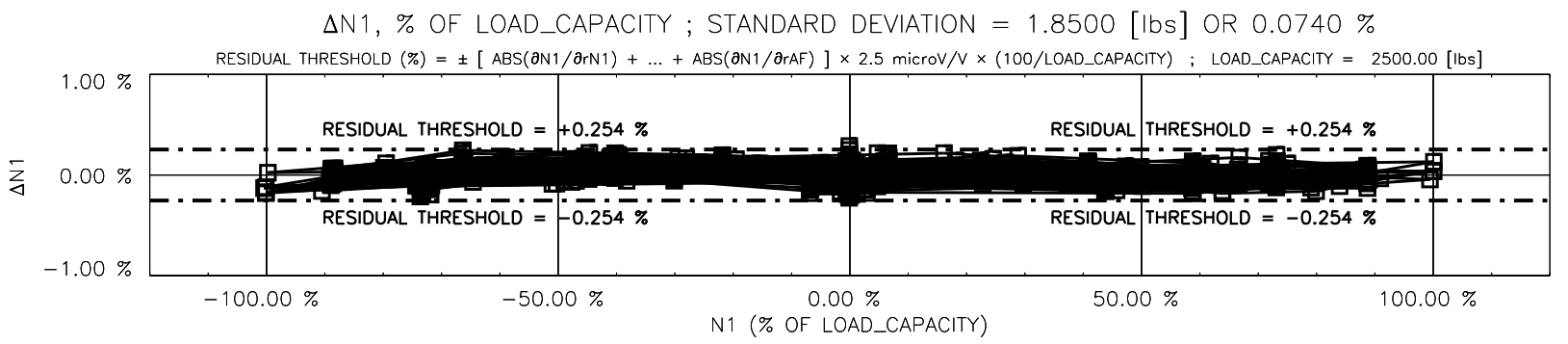

Fig. 7 Load residuals of the forward normal force $N 1$ of the original MC60E calibration data after having been processed in force balance format using the Non-Iterative Method.

This value matches the value that is computed by using the Iterative Method in combination with Load Iteration Method A (see Fig. 4). In addition, residuals plotted in Fig. 4 for the Iterative Method show excellent 
qualitative and quantitative agreement with residuals plotted in Fig. 7 for the Non-Iterative Method. This observation can be supported by comparing the corresponding standard deviations of the residuals. The standard deviation of the residuals shown in Fig. 4 equals $0.0737 \%$. Similarly, the standard deviation of the residuals shown in Fig. 7 equals $0.0740 \%$.

Figure 8 shows results for the total normal force $N F$ after the Non-Iterative Method was used to process the data. In this case, the residual threshold is computed to be $\pm 0.263 \%$.

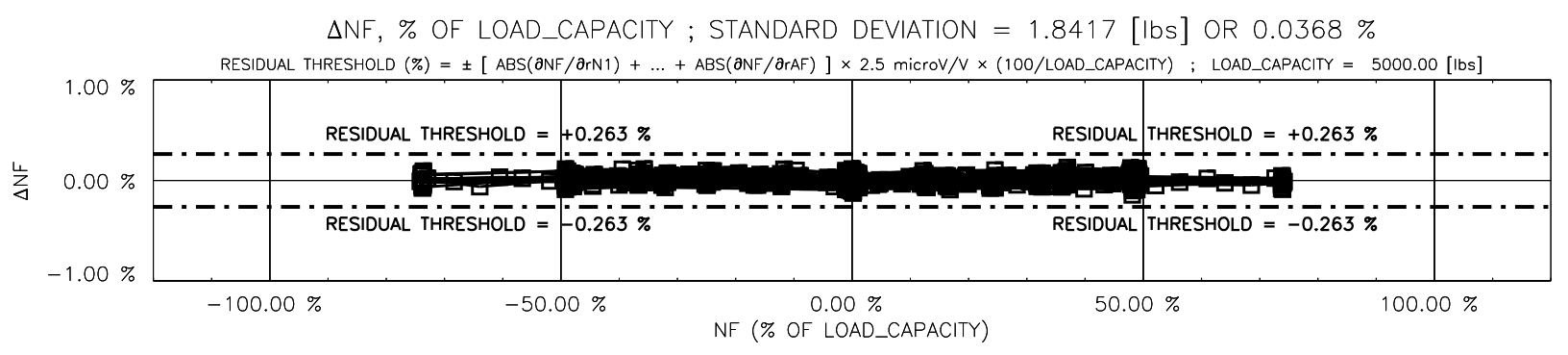

Fig. 8 Load residuals of the total normal force $N F$ of the original MC60E calibration data after having been processed in direct-read format using the Non-Iterative Method.

As expected, it matches the value that is computed by using the Iterative Method in combination with Load Iteration Method A (see Fig. 6). Again, residuals plotted in Fig. 6 for the Iterative Method show excellent qualitative and quantitative agreement with residuals plotted in Fig. 8 for the Non-Iterative Method if the residual plots themselves and the standard deviations of the residuals are compared.

\section{Summary and Conclusions}

A new universally applicable gage output and load residual threshold definition was presented that may be used for a more realistic evaluation of the predictive capability of regression models of wind tunnel strain-gage balance data. The new threshold definition has the advantage that it takes the load resolution capability of each balance gage into account. It can be applied to all known gage output and load formats that are used in the aerospace testing community. In addition, the new definition works with both the Iterative Method and Non-Iterative Method that are used to analyze and process balance data.

The new threshold definition uses an empirical constant of either $2.5 \mathrm{microV} / \mathrm{V}$ or $0.5 \mathrm{microV} / \mathrm{V}$ to assess output residuals of balance data. In addition, corresponding load residual thresholds are obtained by computing the product of the empirical constant with the sum of the absolute values of all first partial derivatives of a load component. Data from the machine calibration of a six-component force balance was used to illustrate the application of the new universal output and load residual threshold definitions.

\section{Acknowledgements}

The authors want to thank Bob Gisler of NASA Ames Research Center for his critical and constructive review of the final manuscript. The work reported in this paper was supported by the Wind Tunnel Division at NASA Ames Research Center under contract NNA16BD26C.

\section{References}

${ }^{1}$ AIAA/GTTC Internal Balance Technology Working Group, Recommended Practice, Calibration and Use of Internal Strain-Gage Balances with Application to Wind Tunnel Testing, AIAA R-091-2003, published by the American Institute of Aeronautics and Astronautics, Reston, Virginia, 2003, pp. 18-19.

${ }^{2}$ Ulbrich, N. and Volden, T., "Application of a New Calibration Analysis Process to the MK-III-C Balance," AIAA 2006-0517, paper presented at the 44th AIAA Aerospace Sciences Meeting and Exhibit, Reno, Nevada, January 2006, p. 3, pp. 9-12.

${ }^{3}$ Ulbrich, N., "Comparison of Iterative and Non-Iterative Strain-Gage Balance Load Calculation Methods," AIAA 2010-4202, paper presented at the 27th AIAA Aerodynamic Measurement Technology and 
Ground Testing Conference, Chicago, Illinois, June/July 2010.

${ }^{4}$ Ulbrich, N., and Volden, T., "A New Load Residual Threshold Definition for the Evaluation of Wind Tunnel Strain-Gage Balance Data," AIAA 2016-1538, paper presented at the 54th AIAA Aerospace Sciences Meeting, San Diego, California, January 2016.

${ }^{5}$ Ulbrich, N., "Assessment of the Uniqueness of Wind Tunnel Strain-Gage Balance Load Predictions," AIAA 2016-4157, paper presented at the 32nd AIAA Aerodynamic Measurement Technology and Ground Testing Conference, Washington, D.C., June 2016.

${ }^{6}$ BALFIT - Software Tool for the Regression Analysis of Multivariate Data - Version 3.01.21, distributed by the Software Release Authority, Technology Partnerships Office, NASA Ames Research Center, Moffett Field, California, December 2014.

${ }^{7}$ Ulbrich, N., and Bader, J., "Analysis of Sting Balance Calibration Data Using Optimized Regression Models," AIAA 2009-5372, paper presented at the 45th AIAA/ASME/SAE/ASEE Joint Propulsion Conference, Denver, Colorado, August 2009, pp. 18-21. 